\title{
Predicting the Amount of Coke Deposition on Catalyst through Image Analysis and Soft Computing
}

\author{
Jingqiong Zhang ${ }^{1}$, Wenbiao Zhang ${ }^{1}$, Yuting $\mathrm{He}^{1}$, Yong Yan ${ }^{2,1^{*}}$ \\ ${ }^{1}$ School of Control and Computer Engineering, North China Electric Power University, Beijing, China \\ ${ }^{2}$ School of Engineering and Digital Arts, University of Kent, Kent CT2 7NT, UK \\ jqzhang@ncepu.edu.cn,wbzhang@ncepu.edu.cn, hyt12200@163.com,y.yan@kent.ac.uk
}

\begin{abstract}
The amount of coke deposition on catalyst is one of the most important indexes of catalytic property and service life. As a result, it is essential to measure the amount of coke deposition and analyze the active state of catalyst during the continuous production process. This paper proposes a new method to predict the amount of coke deposition on catalyst based on image analysis and soft computing. An image acquisition system consisting of a flatbed scanner and an opaque cover is used to obtain catalyst images. After imaging processing and feature extraction, twelve effective features are selected and two best feature sets are determined by the prediction tests. A Neural Network optimized by particle swarm optimization algorithm is used to establish the prediction model of the coke amount based on various datasets. The root mean square error (RMSE) of the prediction values are all below 0.021 and the coefficient of determination $\mathbf{R}^{2}$, for the model are all above $\mathbf{7 8 . 7 1 \%}$. Therefore, a feasible, effective and precise method is demonstrated, which may be applied to realize the real-time measurement of coke deposition based on on-line sampling and fast image analysis.
\end{abstract}

Keywords - coke deposition; catalyst; image analysis; feature extraction; PSO-BP

\section{Introduction}

In the catalyst based continuous production process, one of the key issues to be addressed is the automatic control of the multiphase flow reactor. The selectivity and efficiency of the catalytic system have been improved significantly through the effective control of the multiphase flow reactors [1]. Furthermore, the real-time monitoring of catalyst activity has a significant effect on the control of the reactor.

Catalyst deactivation is mainly due to coke deposition in response of high temperature, e.g. in the processes of FCC (fluid catalytic cracking), MTO (methanol to olefins), GTO (natural gas to olefins) and MTG (methanol to gasoline) [2-4]. Catalyst pellets suffer from the accumulation of coke on their surfaces and mostly in the pore space as the reaction progresses. When the coke gradually grows inside the pore, the active sites of the catalyst are blocked and the mass transfer into the pore is damaged. Finally, it leads to coking deactivation of the catalyst. As a result, the activity and selectivity of the catalyst will be inhibited due to the growing coke deposition, and in the same time the product yield will decrease. However, the coke deposition on the catalyst is often a reversible process and the coked catalyst can be regenerated by burning-off with oxygen in the regenerator. Besides, the coke deposition will also affect the residence time of the reaction, the regenerator temperature and so on [5-7]. Therefore, it is important to measure the amount of coke deposition on catalyst.

The term coke deposition on catalyst is referred to a variety of carbonaceous deposition, mainly hydrocarbon in industry. In general, samples analysis and model-based estimation are the two dominant methods for the measurement of coke deposition [8]. The method of samples analysis quantifies coke 
deposition by sampling catalyst from the reactors and analyzing carbon-element contents of the samples. Among them, TGA (Thermogravimetric Analysis) is a traditional method for chemical analysis based on the evaluation of the samples' thermal behavior. The carbon content of the catalyst samples is determined by the weight or mass ratios before and after heating [9]. As a well-established method, TGA is usually applied in laboratory-scale measurement. However, the processes of sampling, heating and weighing often take a lot of time, leading to a lag between the state of coke deposition and the control of the reactor. In addition, there are systematic errors in the TGA method which may be caused by the complex operations, e.g. the judgment of the initial weightlessness position of the TG curves. The existence of these problems has seriously affected its efficient applications in industrial production processes.

Combustion-infrared detection can also be used to quantify carbon formation regarding the coke deposition on catalyst. By feeding oxygen and burning catalyst samples under high temperature, the carbon in the coke deposition is converted into carbon dioxide. As carbon dioxide is a strong absorber of the infrared wave with $4260 \mathrm{~nm}$ wavelength, the carbon content can be determined using an infrared gas analyzer which measures the intensity of the infrared spectra of carbon dioxide evolved from the samples [10]. Similarly, TPO (Temperature-programmed oxidation) method obtains the amount of coke deposition based on samples by burning and elementary analysis of effluent gases. Furthermore, there are other methods like TPO combined with either gas chromatography or gravimetric gas analysis to measure coke deposition by determining the carbon content of catalyst samples. These methods require contact with the samples and cannot meet the demands of real-time measurement for industrial applications [11, 12].

Model-based estimation method is a kind of 'soft' measurement method. Through feature analysis, a predicting model is developed between the features and the amount of coke deposition on catalyst to realize real-time measurement. A new acoustic measurement method has been proposed by Tang et al $[13,14]$. In the process of catalytic reaction, catalyst pellets in the fluidized bed reactor hit the internal wall of the reactor and generate acoustic emission signals. In combination with the analysis of the power spectrum of the signals, the amount of coke deposition on catalyst can be predicted using the characteristic parameters of the acoustic emission signals. This on-line measurement method can overcome the shortcomings of traditional sampling analysis which entails the delay in the control of the reactor. However, this method does not work in a fixed bed reactor where catalyst pellets usually stay still.

On account of the limitations on all existing methods mentioned above, the aim of this paper is to propose a novel method to measure the amount of coke deposition on catalyst through image analysis. Some preliminary results of this method was reported at the 2015 IEEE International Conference on Imaging Systems \& Techniques [15]. In this paper, we present in detail the image acquisition and processing system, extract the effective features from the images of the catalyst pellets and establish a model to predict the coke amount through soft computing. Finally, the prediction model based on Back Propagation neural network with Particle Swarm Optimization (PSO-BP) algorithm is established and validated using various datasets.

\section{Methodology}

\subsection{Image acquisition system}

In order to ensure the stability of the shooting angle, exposure time and visual field area for each set of images, a flatbed scanner with a matrix CCD image sensor is used instead of ordinary CCD cameras. 
In addition, the light source of an image acquisition system should be uniform and stable. A shading system based on a flatbed scanner and an opaque cover, as shown in Fig. 1, is developed to provide a invariable background illumination that can avoid the different lighting conditions for each set of images. The scanner can scan the A4 format area $(216 \mathrm{~mm} \times 311 \mathrm{~mm})$ during which substantial catalyst pellets were scanned in one go to meet the statistical significance of the acquired data. At the same time, the height of the opaque cover from the scan platform is adjusted to $5 \mathrm{~cm}$ so as to easily distinguish the catalyst pellets from the background. Besides, the colour of the cover is white in order to make a relatively bright background in contrast with dark catalyst pellets. In our research, the resolution of the scanner is selected as $4800 \mathrm{dpi} \times 4800 \mathrm{dpi}$ and each pixel size is approximately $45 \mu \mathrm{m} \times 65 \mu \mathrm{m}$ after taking the size and the gray value of the catalyst pellets into account.

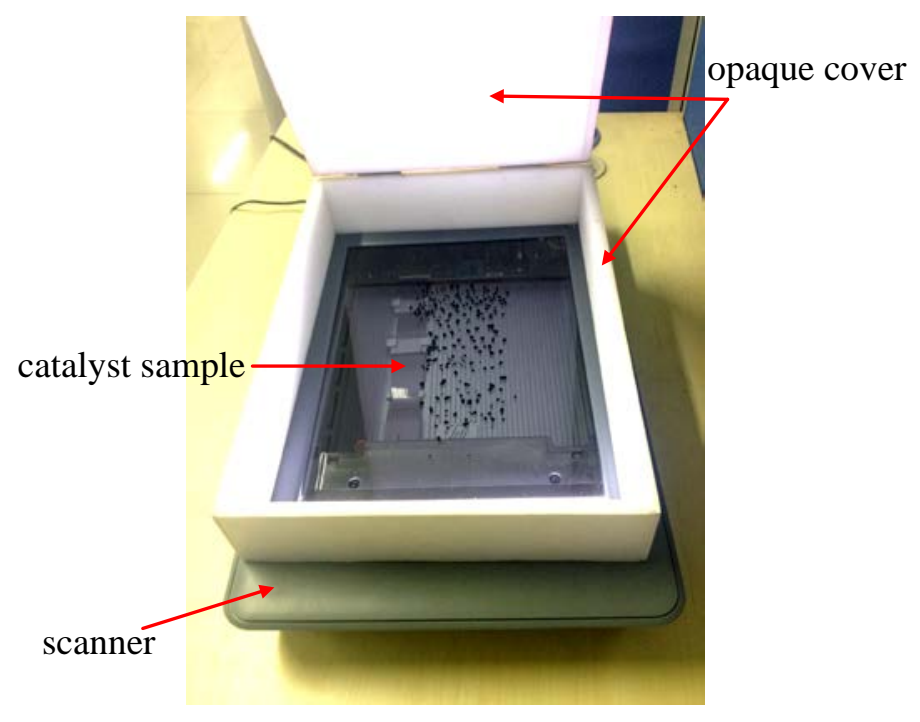

Fig. 1. Image acquisition system.

\subsection{Sample preparation}

A kind of molecular sieve solid catalyst in MTO (methanol to olefins) process was used in this study. The samples were collected sequentially from the coked catalyst over a period of time in the same MTO reaction process. As a result, eight types of coked catalyst pellets were obtained from the same reactor with the deepening coke deposition corresponding to different coking periods. The samples were divided into two sets. One set was measured as the standard coke deposition amount of the catalyst by TGA. As for the other set, the catalyst images were taken using the scanner. In view of the fact that the coking process of catalyst mainly takes place in the internal pores, simple physical treatment of the samples was conducted. The samples were cut into smaller pellets in order to get more internal information about the catalyst. After the treatment, the majority of the catalyst pellets were in the range from $1 \mathrm{~mm}$ to $5 \mathrm{~mm}$ in size. Table 1 gives the actual coke amount of eight types of catalyst pellets by TGA method.

TABLE 1 Coke Amount of Eight Types of Catalyst by TGA Method

\begin{tabular}{|c|cccccccc|}
\hline Category & $\# 1$ & $\# 2$ & $\# 3$ & $\# 4$ & $\# 5$ & $\# 6$ & $\# 7$ & $\# 8$ \\
\hline Coke amount (\%) & 30.316 & 29.622 & 28.038 & 27.157 & 22.549 & 20.067 & 0.448 & 0.418 \\
\hline
\end{tabular}

\subsection{Image processing}

Fig. 2(a) is the image of seriously coked catalyst with a high level of coke deposition and its gray scale histogram is shown in Fig. 2(b) with two distinct peaks. One of the peak is below 50 owing to the 
seriously coked catalyst regions and the other peak is around 100 corresponding to the background area. Fig. 2(c) shows the image of slightly coked catalyst with a small amount of coke deposition and the corresponding gray scale histogram is shown in Fig. 2(d). Similar to Fig. 2(b), there are also two peaks in Fig. 2(d). One above 200 is generated from slightly coked catalyst regions and the other one around 100 corresponds to the background area. The obvious difference between the foreground (catalyst pellets) and the background regions, with the stability of the lighting conditions, indicates the image acquisition system is feasible for the intended application.
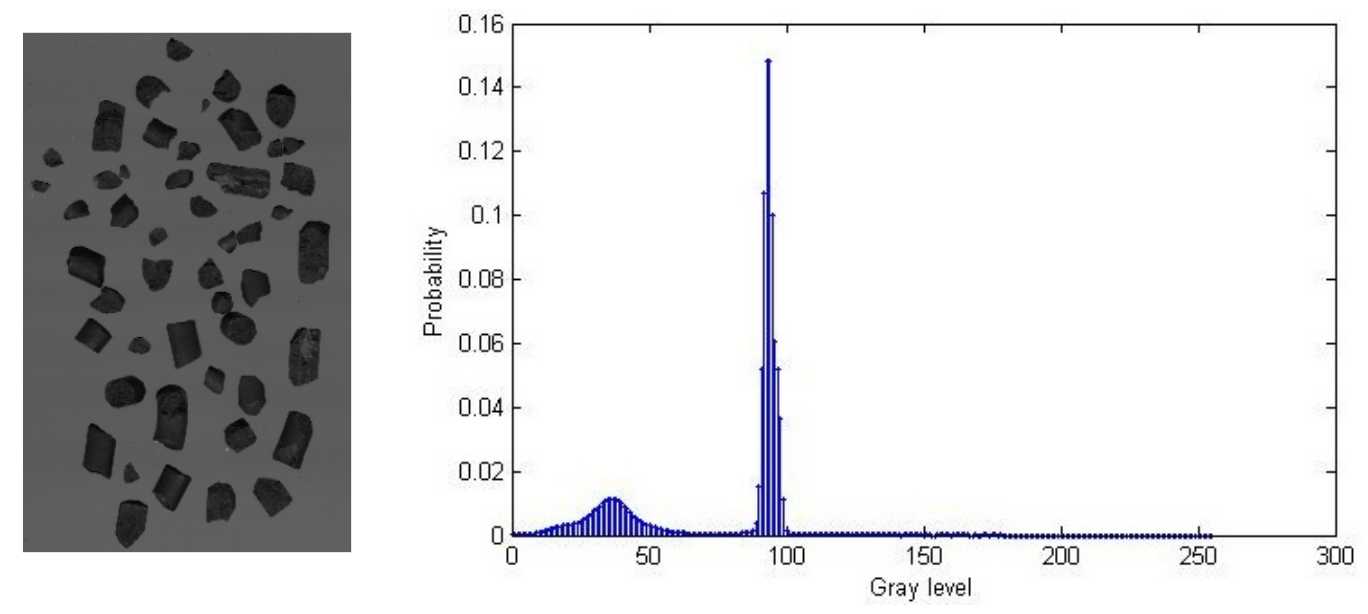

(a) Image of seriously coked catalyst.

(b) Normalized histogram of image (a).

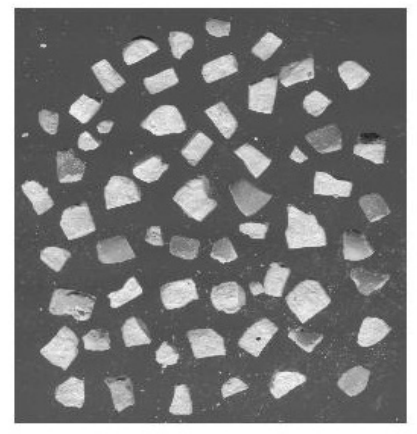

(c) Image of slightly coked catalyst.

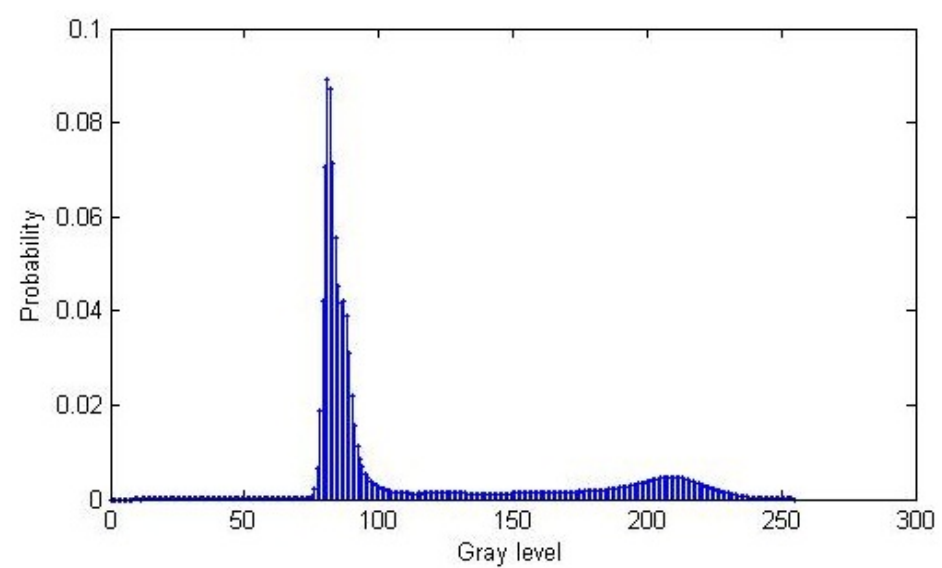

(d) Normalized histogram of image (c).

Fig. 2. Catalyst images and their gray-scale histograms.

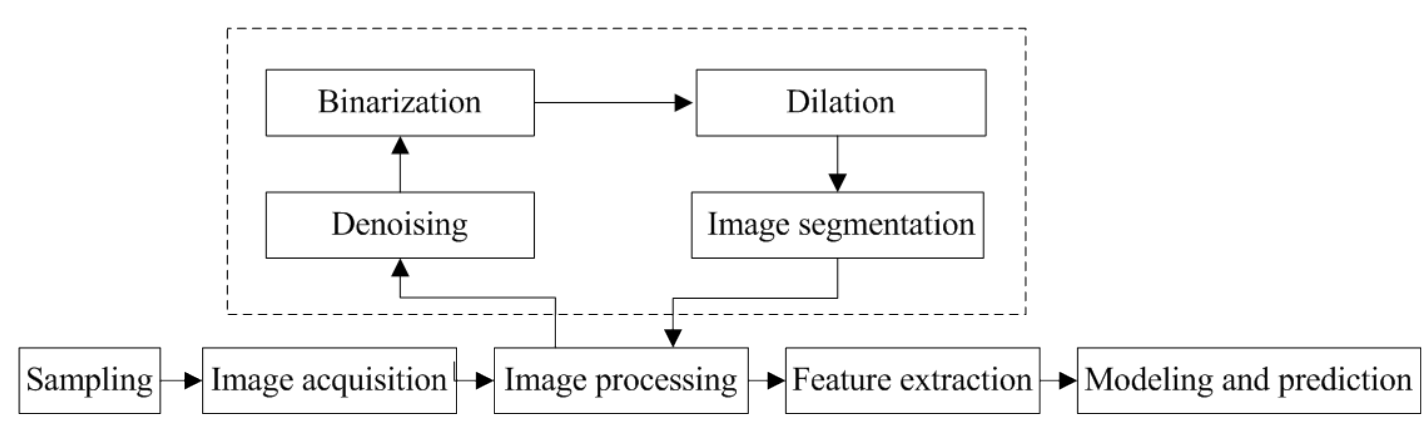

Fig. 3. Flow chart of the proposed method. 
The main logical steps of the proposed method, including the key image processing procedure, are shown in Fig.3. Since sometimes there may be dirt or dust on the scan platform, area thresholding and morphological filtering are used to remove the unwanted artifacts in the image. After denoising, the task of image processing is to segment catalyst pellets by dividing the image into foreground region (catalyst pellets) and background. According to the different gray values between the catalyst pellets and the background based on the gray scale histograms, the Otsu method [16] is used to convert the colour images into binary images. The optimal threshold is selected on the basis of maximizing inter-class variance. As shown in Fig. 4(c) and 4(d), some small interior holes appear in the results of the binarization after the application of Otsu method. These holes originate from the direct contact of the samples with the scan platform, corresponding to the highlight regions of catalyst pellets. Therefore, the next step is to fill these small interior holes by using a series of dilation. The binary images after region growing are shown in Fig. 4(e) and 4(f), in which “1” (logical value) stands for the catalyst pellets and "0" (logical value) denotes the background. Finally, the segmentations of catalyst pellets are accomplished after multiplying the original images by the binary images. In view of the fact that the gray values of some seriously coked regions are approximately equal to 0 (black), they are presented in white background, while the slightly coked catalyst pellets are presented in black background, as shown in Fig. 4(g) and 4(h).

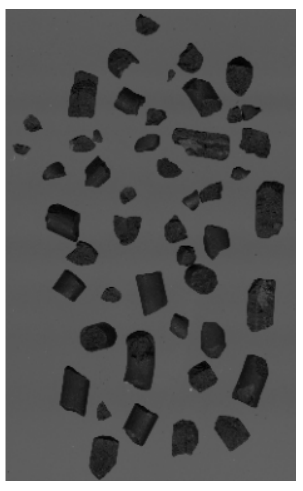

(a) Original image of

seriously coked catalyst.

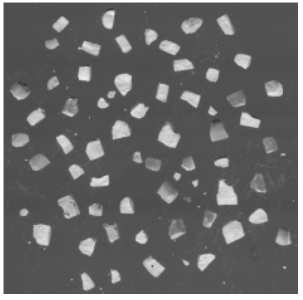

(b) Original image of

slightly coked catalyst.

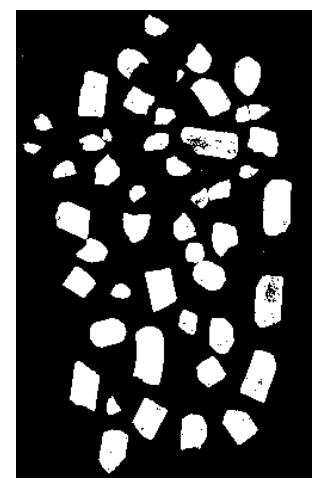

(c) Segmented image of seriously coked catalyst using Otsu method.

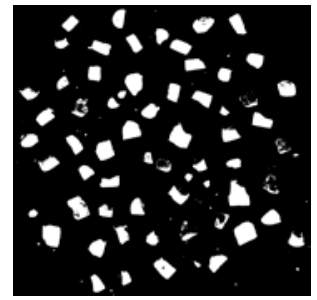

(d) Segmented image of slightly

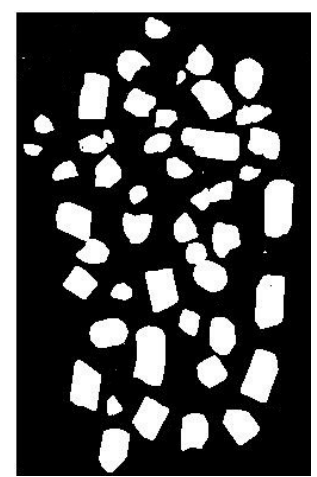

(e) Binary image of seriously coked catalyst after dilation.

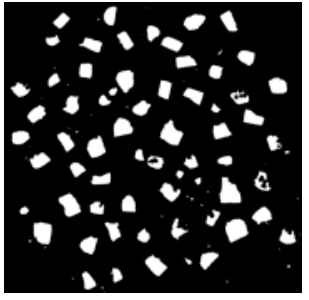

(f) Binary image of slightly coked catalyst after dilation.

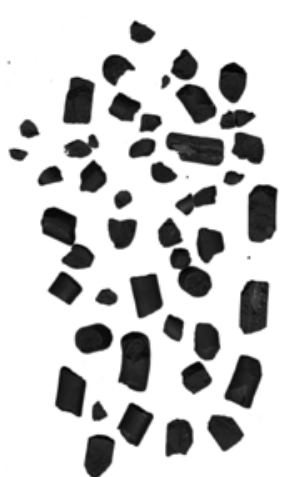

(g) Final segmented image of seriously coked catalyst.

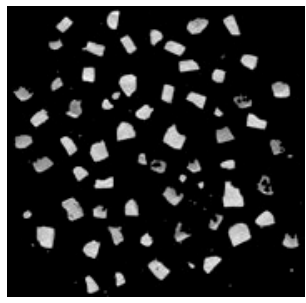

(h) Final segmented image of slightly coked catalyst.

Fig. 4. Original and processed catalyst images.

\subsection{Extraction of colour features}

It was observed that very often the catalyst pellets change from creamy white to black due to the accumulation of coke deposition on them in many reaction processes. Besides, it has been proved that the size of catalyst pellets remains roughly unchanged as the coke is gradually deposited [14]. Therefore, effective colour features should be used to reflect the state of the catalyst and measure the 
amount of coke deposition based on the analysis of catalyst pellets images during different periods of the reaction.

Colour features play an important part in the application of image classification. Stricker and Orengo [15] proposed that the effective color features were the low moments of the colour components derived from a colour image. The first moment is the mean value of the image. The second moment stores the variance of the image whilst the third moment stands for the skewness of the image $[17,18]$. They are calculated from the following mathematical expressions:

$$
\begin{gathered}
m=\sum_{i=0}^{L-1} x_{i} p\left(x_{i}\right) \\
\sigma=\sqrt{\sum_{i=0}^{L-1}\left(x_{i}-m\right)^{2} p\left(x_{i}\right)} \\
\mu=\sqrt[3]{\sum_{i=0}^{L-1}\left(x_{i}-m\right)^{3} p\left(x_{i}\right)}
\end{gathered}
$$

where $m, \sigma$ and $\mu$ are the three low moments of the colour image, respectively. $x_{i}$ denotes the $i^{\text {th }}$ intensity levels in the colour distribution of the image and $p\left(x_{i}\right), i=0,1,2, \ldots, L-1$, represents the corresponding probability histogram in which $L$ is the number of distinct intensity levels.

In addition, for the randomness of the colour components, other two features, entropy and energy, are also calculated, respectively, from the following equations:

$$
\begin{gathered}
E_{t}=-\sum_{i=0}^{L-1} p\left(x_{i}\right) \log _{2} p\left(x_{i}\right) \\
E_{n}=\sum_{i=0}^{L-1} p\left(x_{i}\right)^{2}
\end{gathered}
$$

where $E_{t}$ denotes entropy and $E_{n}$ stands for energy.

In general, the analysis of the image intensity layer by calculating the image gray value is regarded as a simple method for colour features extraction. However, in this case, the image colour information is completely ignored. Another common approach is to separate the red, green, and blue (RGB) layers from the colour image and then analyze them as three independent ones. Such an approach considers some colour information, but the relationships between different colour layers, containing more color details, are overlooked [19, 20].

Considered to be more fit for human perception, the HSI colour model consisting of hue, saturation, intensity is applied to show the correlations between different colour layers in order to make up for this deficiency. The normalization of the three RGB components is achieved by dividing RGB colour values by 255 and all values are subsequently changed from $[0,255]$ to $[0,1]$ with MAX denoting the maximum and MIN the minimum of the colour values. The transformation of the colour components from the RGB colour model to the HSI colour model is achieved as follows: 


$$
\begin{aligned}
& H=\left\{\begin{array}{c}
\frac{G-B}{M A X-M I N} \times 60, \quad(R=M A X) \\
\left(2+\frac{B-R}{M A X-M I N}\right) \times 60, \quad(G=M A X) \\
\left(4+\frac{R-G}{M A X-M I N}\right) \times 60, \quad(B=M A X)
\end{array}\right. \\
& S=\frac{M A X-M I N}{M A X} \\
& I=\frac{1}{3}(R+G+B)
\end{aligned}
$$

Typical results of colour features extraction from the segmented images of the eight types of catalyst samples are shown in Fig. 5. The standard amount of coke deposition on catalyst is measured by TGA. Fig. 5(a) shows the changes of R, G, B and gray mean features with the accumulation of coke deposition on catalyst. Fig. 5(b) depicts the trends of the three low moments (mean, variance, skewness) of the gray component as the coke deposition deepens.

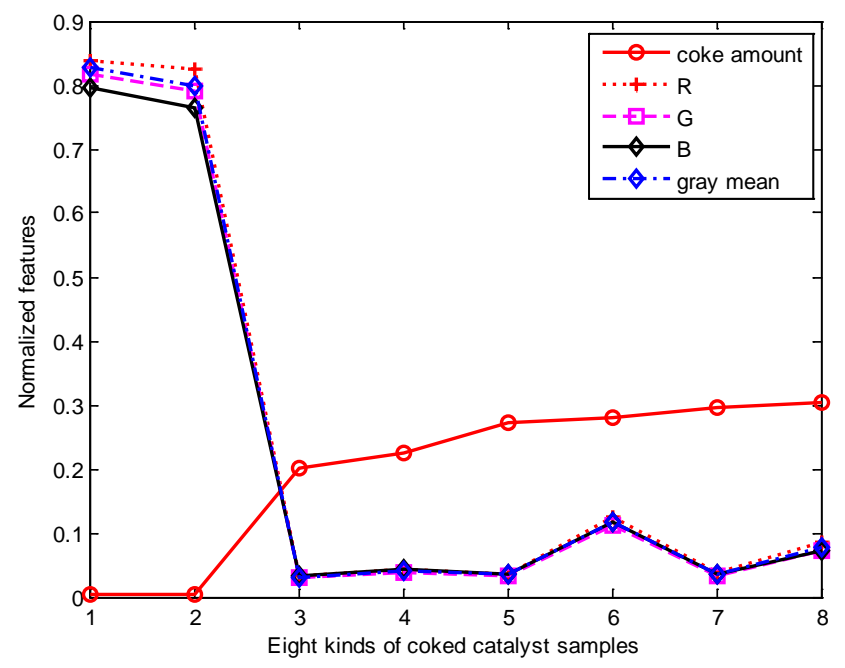

(a) R, G, B and gray mean.

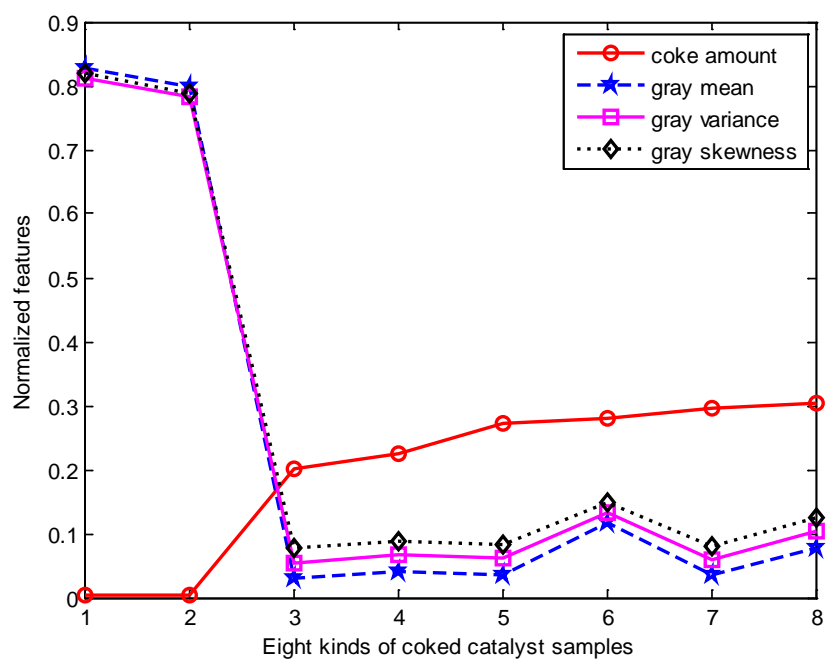


(b) Gray mean, gray variance and gray skewness.

Fig. 5. Comparison of some colour features and the coke amount.

As mentioned above, the extraction of colour features can be conducted in different layers including R, G, B and gray layers. Note that the values of R, G, B and gray components are almost the same in the segmented catalyst images, as their mean values are shown in Fig. 5(a). It is necessary to determine the colour features from which of the R, G, B and gray layers are more effective. In addition, in view of the similar trends of the three low gray moments (mean, variance, skewness) as shown in Fig. 5(b), the mean values of the colour components are calculated for evaluating the discriminability in different layers as representative. As a result, three features, including the mean value, entropy and energy, are calculated in the R, G, B and gray layers to evaluate the discriminability of colour features in different colour layers. In this research the ratio of the inter-class distance to the intra-class distance represented by the data variance is used to define a discriminability index:

$$
D^{k}=\frac{D_{\text {inter }}{ }^{k}}{D_{\text {intra }}}=\frac{S^{2}\left(\operatorname{mean}\left(V_{i}^{k}\right)\right)}{\sum_{i=1}^{8} S^{2}\left(V_{i}^{k}\right)}
$$

where $V_{i}^{k}$ is the $k^{\text {th }}$ colour components of the $i^{\text {th }}$ image of the eight types of catalyst samples and mean $\left(V_{i}^{k}\right)$ is the average of $V_{i}^{k}$, and $S^{2}$ stands for the variance of the respective variable. Besides, $D_{\text {inter }}{ }^{k}$ and $D_{\text {intra }}{ }^{k}$ are the inter-class and intra-class distances of $k^{\text {th }}$ colour components, respectively, for the eight types of catalyst samples. $D^{k}$ is referred to as discriminability of the $k^{\text {th }}$ colour components. All data should be normalized before $D^{k}$ is calculated from Eq. (9). The larger the value of $D$ is, the greater discriminability denotes.

TABLE 2 Discriminability Index of Three Colour Features in Different Colour Layers

\begin{tabular}{|c|c|cccc|}
\hline \multirow{2}{*}{ Colour feature } & \multirow{2}{*}{$\boldsymbol{D}$} & \multicolumn{4}{|c|}{ Different colour layers } \\
\cline { 3 - 6 } & & $\mathbf{R}$ & $\mathbf{G}$ & $\mathbf{B}$ & Gray \\
\hline Mean & $\boldsymbol{D}^{\mathbf{1}}$ & 0.616 & 0.589 & 0.736 & 0.762 \\
Entropy & $\boldsymbol{D}^{\mathbf{2}}$ & 0.341 & 0.338 & 0.394 & 0.406 \\
Energy & $\boldsymbol{D}^{\mathbf{3}}$ & 0.347 & 0.288 & 0.446 & 0.440 \\
\hline Sum of & $\mathbf{D}^{\mathbf{k}}$ & $\mathbf{1 . 3 0 4}$ & $\mathbf{1 . 2 1 5}$ & $\mathbf{1 . 5 7 6}$ & $\mathbf{1 . 6 0 8}$ \\
\hline
\end{tabular}

Table 2 summarizes the results of discriminability analysis. By comparing the sum of $D^{k}(k=1,2,3)$ corresponding to the three colour features (mean, entropy, energy) in R, G, B and gray layers respectively, the maximum one belongs to the most effective layer. Since the sum of $D^{k}(k=1,2,3)$ in gray layer is the maximum of all results, the gray layer is regarded as the most effective one. Furthermore, the values of $D^{1}$ in all layers are significantly greater than $D^{2}$ and $D^{3}$ as shown in Table 2 . It means the mean feature is more effective than entropy and energy. Therefore, it can be concluded that the extraction of colour features should be conducted in gray layer and the gray mean feature is most effective to predict the coke amount with the greatest discriminability.

As a result, five gray features (mean, variance, skewness, entropy and energy) were extracted from the gray layer. In total six features (R, G, B, H, S, I) were obtained from the RGB and HSI colour models, with the results of feature extraction from the eight types of catalyst samples shown in Fig. 5 and Fig. 6. Some results of colour features extraction are also shown in Fig. 5. The relationships between the other five features (H, S, I, gray entropy and gray energy) and the coke amount are plotted 
in Fig. 6. The strongest similarities between the curve of $\mathrm{H}$ value and the level of coke deposition are evident. In summary, $\mathrm{H}$ and gray mean values are regarded as indicators of the coke amount.

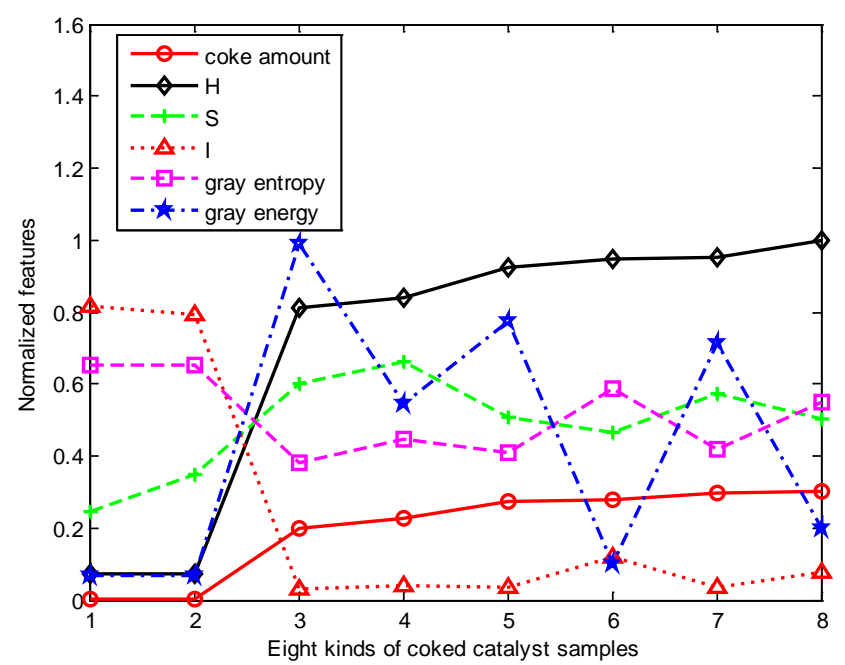

Fig. 6. Comparisons of H, S, I, gray entropy and gray energy features and the coke amount.

\subsection{Extraction of texture features}

As one of the important characteristics, textural information is useful for in image classification by analyzing the objects or the regions of interest in an image [21]. Considering all the above eleven colour features are extracted from the colour components based on statistical moments of intensity histograms regardless of the spatial distribution of pixels with respect to each other, texture features are also extracted from the catalyst images in our study. Texture features can help to find more characteristics, for instance the smoothness of coked catalyst pellets, and improve the prediction results of the coke amount.

In order to describe texture variations, one way to compute the relative positions of pixels in an image or region is to use the Gray Level Co-occurrence Matrix (GLCM). Haralick, Shanmugam and Dinstein [21] put forward the views of the image texture features for image classification in 1973. As a classical texture analysis approach, GLCM reflect the gray spatial correlation of pixels in the segmented catalyst pellets regions. In this research, six texture features of GLCM were extracted: maxprobability, contrast, correlation, energy, homogeneity and entropy. Maxprobability feature describes the strongest response in the GLCM matrix. Contrast is an important feature to characterize the local variations by calculating the gray intensity contrast between the pixels of relative positions. The linear dependencies of gray values between the pixels in relative positions are computed by correlation feature. Energy is an index of uniformity which measures the constancy of gray values distribution in an image or a region. By comparing the gray values distribution of the pixels on the diagonal direction and off the diagonal direction of the GLCM, homogeneity feature is determined. Entropy is used to detect the disorder or textural irregularity of an image or a region. Detailed information about the calculation of GLCM and the mathematical expressions of these texture features can be found in the references [22, 23]. As a result, seventeen features including colour and texture features could be obtained from each catalyst pellet region, which are R, G, B, H, S, I, Gray features (gray mean, gray variance, gray skewness, gray energy and gray entropy) and GLCM features (maxprobability, contrast, correlation, energy, homogeneity, entropy). 


\section{Results and discussion}

\subsection{Image database}

The image database was collected by scanning the eight types of coked catalyst samples mentioned in Part 2.2. The images of twenty-five catalyst pellets were segmented to extract seventeen features as mentioned above for each type of catalyst sample. As a result, $3400(25 * 8 * 17)$ features were obtained from the total of $200(25 * 8)$ catalyst pellets.

\subsection{Feature selection}

After feature extraction, the next step is to select the effective features which obtain the useful information to predict the coke amount. In order to find the best feature sets to establish the prediction model, testing method was used to train the model and select the features according to the prediction results. The inputs of the prediction model were different combinations of features extracted from catalyst pellets images, and the outputs were the actual coke amount of catalyst pellets measured by TGA method given by Table 1. In detail, seventeen features as mentioned above were extracted from the image database and then the corresponding feature datasets which were divided into two parts, the training dataset and the testing dataset, could be obtained from different combinations of these features. Before the model training, all features should be normalized to [0, 1]. And then, the training dataset was used to establish the prediction model of the image features and actual coke amount based on PSO-BP algorithm. Finally, the testing dataset was inputted to the model, RMSE (Root Mean Square Error) and the coefficient of determination $\mathrm{R}^{2}$ of prediction results could be obtained to evaluate the prediction performance of the model. The calculation formulas of RMSE and $\mathrm{R}^{2}$ are given as follows, and when RMSE is smaller and $\mathrm{R}^{2}$ is closer to 1 , the prediction model performs better.

$$
\begin{gathered}
R M S E=\sqrt{\frac{\sum_{i=1}^{m}\left(\hat{y}_{i}-y_{i}\right)^{2}}{m}} \\
R^{2}=1-\frac{\sum_{i=1}^{m}\left(\hat{y}_{i}-y_{i}\right)^{2}}{\sum_{i=1}^{m}\left(\bar{y}-y_{i}\right)^{2}}
\end{gathered}
$$

where $y_{i}$ is the actual coke amount of the $i^{\text {th }}$ catalyst pellet, $\hat{y}_{i}$ is the predicted coke amount of the $i^{\text {th }}$ catalyst pellet, $\bar{y}$ is the average actual coke amount of all catalyst pellets and $i=1,2,3, \ldots, m$, in which $m$ denotes the number of catalyst pellets.

In machine learning $30 \%-50 \%$ of a database is usually chosen as the testing dataset while the remaining data is used for training. In this section, two datasets named Random 7 and Random 10 were selected and chosen as datasets. Random 7 dataset consists of $56(7 * 8)$ catalyst pellets composed by randomly selected 7 pellets of each type as the testing dataset and the left $144(18 * 8)$ pellets as the training dataset. Besides, Random 10 dataset consists of $80(10 * 8)$ catalyst pellets composed by randomly selected 10 pellets of each type as the testing dataset and the left $120(15 * 8)$ pellets as the training dataset.

The conclusion in Part 2.4 illustrates that among all colour features, $\mathrm{H}$ and gray mean value own the good ability to predict coke amount. The results of RMSE and $\mathrm{R}^{2}$ of A1, A2, A3, where gray mean, $\mathrm{H}$ and their combination were selected as feature sets correspondingly are shown in Table 3. The prediction effects demonstrate the availability of these two features in predicting the coke amount. And 
then on the basis of gray mean and $\mathrm{H}$ features, other fifteen features were added to the feature sets one at a time and the features were inputted to the prediction model established by PSO-BP algorithm respectively in order to test prediction effects. The effective ones could be selected by judging the RMSE and $\mathrm{R}^{2}$ of the prediction results as shown in Table 3. Compared with the prediction effects of the combination of gray mean and $\mathrm{H}$ features, those feature sets with worse prediction performance than A3 should be eliminated. That is to say A11, A13, A14, A16 and A17 should be excluded. Accordingly, five features, namely gray skewness, gray entropy, maxprobability, correlation and energy, were eliminated.

As a result, nine colour features and three texture features were picked out as effective features, which are R, G, B, H, S, I, gray mean, gray variance, gray energy, contrast, homogeneity and entropy.

TABLE 3 Predicting Results of H, Gray Mean and Other Feature Sets

\begin{tabular}{|c|c|c|c|c|c|}
\hline & \multirow{2}{*}{ Feature set } & \multicolumn{2}{|c|}{ Random 7} & \multicolumn{2}{c|}{ Random 10} \\
\cline { 2 - 5 } & gMSE & $\mathrm{R}^{2}$ & $\mathrm{RMSE}$ & $\mathrm{R}^{2}$ \\
\hline A1 & gray mean & 0.0278 & $94.40 \%$ & 0.0261 & $95.04 \%$ \\
A2 & $\mathrm{H}$ & 0.0327 & $92.45 \%$ & 0.0355 & $91.55 \%$ \\
A3 & gray mean, H & 0.0284 & $93.99 \%$ & 0.0273 & $94.52 \%$ \\
A4 & gray mean, H, R & 0.0220 & $96.51 \%$ & 0.0224 & $96.37 \%$ \\
A5 & gray mean, H, G & 0.0228 & $96.24 \%$ & 0.0209 & $96.85 \%$ \\
A6 & gray mean, H, B & 0.0249 & $95.53 \%$ & 0.0193 & $97.36 \%$ \\
A7 & gray mean, H, S & 0.0263 & $94.97 \%$ & 0.0192 & $97.38 \%$ \\
A8 & gray mean, H, I & 0.0242 & $95.73 \%$ & 0.0218 & $96.59 \%$ \\
A9 & gray mean, H, entropy & 0.0213 & $96.69 \%$ & 0.0233 & $96.00 \%$ \\
A10 & gray mean, H, gray variance & 0.0258 & $95.22 \%$ & 0.0205 & $97.00 \%$ \\
A11 & gray mean, H, gray skewness & 0.0327 & $92.47 \%$ & 0.0289 & $94.11 \%$ \\
A12 & gray mean, H, gray energy & 0.0223 & $96.41 \%$ & 0.0213 & $96.69 \%$ \\
A13 & gray mean, H, gray entropy & 0.0322 & $92.56 \%$ & 0.0364 & $91.28 \%$ \\
A14 & gray mean, H, maxprobability & 0.0285 & $93.93 \%$ & 0.0313 & $92.83 \%$ \\
A15 & gray mean, H, contrast & 0.0247 & $95.61 \%$ & 0.0227 & $96.26 \%$ \\
A16 & gray mean, H, correlation & 0.0464 & $84.24 \%$ & 0.0327 & $92.47 \%$ \\
A17 & gray mean, H, energy & 0.0382 & $89.47 \%$ & 0.0358 & $91.41 \%$ \\
A18 & gray mean, H, homogeneity & 0.0220 & $96.51 \%$ & 0.0234 & $96.04 \%$ \\
\hline
\end{tabular}

It is a usual phenomenon that some correlations lie in the different features, for instance in the R, G, $\mathrm{B}, \mathrm{H}, \mathrm{S}$ and I features. In order to reduce the redundancy of data and improve the prediction results, more simulation tests were conducted to determine which one performs the best among various feature sets. A total of 1586 groups of feature sets were tested by the prediction model, consisting of the different combinations of the randomly selected seven, eight, nine, ten, eleven and twelve features from the twelve effective ones mentioned above. Table 4 gives some excellent feature sets of which the RMSE are below 0.03 and $\mathrm{R}^{2}$ are above $94 \%$ in the simulation tests. Among them, the F1 and F2 feature sets outstand due to the best prediction effect and the less feature number.

TABLE 4 Predicting Effects of Some Excellent Feature Sets

\begin{tabular}{|c|c|c|c|c|c|}
\hline \multirow{2}{*}{ Feature set } & \multicolumn{2}{|c|}{ Random 7 } & \multicolumn{2}{|c|}{ Random 10 } \\
\cline { 3 - 5 } & & RMSE & $\mathrm{R}^{2}$ & RMSE & $\mathrm{R}^{2}$ \\
\hline
\end{tabular}




\begin{tabular}{|c|c|c|c|c|c|}
\hline F1 & $\begin{array}{l}\mathrm{H}, \mathrm{S}, \mathrm{I} \text {, gray mean, gray variance, gray } \\
\text { energy, contrast }\end{array}$ & 0.0172 & $97.85 \%$ & 0.0211 & $96.77 \%$ \\
\hline F2 & $\begin{array}{l}\text { B, H, S, I, gray mean, gray variance, gray } \\
\text { energy, homogeneity, entropy }\end{array}$ & 0.0218 & $96.57 \%$ & 0.0209 & $96.85 \%$ \\
\hline F3 & $\begin{array}{l}\text { R, G, H, S, gray mean, gray variance, } \\
\text { gray energy, homogeneity, entropy }\end{array}$ & 0.0283 & $94.25 \%$ & 0.02 & $97.08 \%$ \\
\hline F4 & $\begin{array}{l}\text { R, G, B, H, S, I, gray mean, gray variance, gray } \\
\text { energy, contrast, homogeneity, entropy }\end{array}$ & 0.0271 & $94.69 \%$ & 0.0221 & $96.49 \%$ \\
\hline F5 & $\begin{array}{l}\text { R, G, B, H, I, gray mean, gray variance, } \\
\text { gray energy, homogeneity }\end{array}$ & 0.0283 & $94.25 \%$ & 0.0284 & $94.21 \%$ \\
\hline F6 & $\begin{array}{l}\text { G, B, S, I, gray mean, gray variance, } \\
\text { gray energy, contrast, entropy }\end{array}$ & 0.0242 & $95.71 \%$ & 0.0280 & $94.30 \%$ \\
\hline F7 & $\begin{array}{l}\text { B, H, S, I, gray mean, gray variance, gray } \\
\text { energy, contrast, homogeneity, entropy }\end{array}$ & 0.0210 & $96.88 \%$ & 0.0249 & $95.47 \%$ \\
\hline
\end{tabular}

Finally, according to the prediction results of different feature sets, two best feature sets have been identified as follows:

F1 H, S, I, gray mean, gray variance, gray energy, contrast;

F2 B, H, S, I, gray mean, gray variance, gray energy, homogeneity, entropy;

\subsection{Prediction model training}

Two popular artificial intelligence algorithms, Neural Network (NN) and Support Vector Machine (SVM), were used to establish the prediction model of the coke amount and the features of catalyst images. A BP neural network is a kind of multilayer feed forward network trained by the error back propagation algorithm. The method calculates the gradient of a loss function with respect to all the weights in the network. The gradient is stored for the optimization method which in turn uses it to update the weights $[24,25]$. In order to improve the prediction results of the model, the particle swarm optimization (PSO) algorithm was applied to optimize BP neural network in this paper. In the implementation of the PSO algorithm, each particle represents a potential solution to an optimization problem and is defined by three parameters to realize the optimization, i.e. position, velocity and fitness values. The velocity determines the direction and distance of the particles' movement whilst the fitness value of each particle is used to evaluate the merits of the particles. PSO is valid for the application to optimize the weights and thresholds of BP network, where each particle represents one weight or threshold. After the PSO optimization, the optimized initial weight and threshold obtained from PSO are assigned to BP network [26]. In this research, the node number of the input layer in the BP network is kept the same as that of the input features and the node number of the output layer is 1 . With respect to the node number of the hidden layer, the learning ability of the BP network will be poor when the node number is too few, but an excessively high number of nodes will increase the training time. In this research, the node number of the hidden layer is set as 35. Moreover, the training times of the BP network are 100 according to the performance of the prediction model. As for the PSO algorithm, the population size of particles is set to 30 . The evolution number is 50 , which is set based on experience and the particle dimension is determined by the number of nodes in each layer in the BP network. After the 
PSO optimization, the optimized initial weight and threshold obtained from PSO are assigned to BP. After datasets training, the output can be acquired as the predicted values. Besides, Support Vector Machine (SVM), which is a supervised learning model in the field of machine learning, typically used for pattern recognition, classification, and regression analysis, was also used to establish the prediction model. In this paper, Lib-SVM toolbox developed by Chih Jen of Taiwan University was used and the best set of two main parameters, penalty parameter (c) and kernel function parameter (g), was ascertained by K-fold Cross Validation (K-CV) method [27].

Comparing the prediction results of these two algorithms shown in Table 5, it comes to the conclusion that both of them show good predicting ability, but the PSO-BP algorithm performs better.

TABLE 5 Comparison of Predicting Effects of PSO-BP and SVM

\begin{tabular}{|c|c|c|c|c|c|}
\hline \multirow{2}{*}{\multicolumn{2}{|c|}{$\begin{array}{ll}\text { Algorithm } & \text { Dataset } \\
\text { Index } & \text { Feature set }\end{array}$}} & \multicolumn{2}{|c|}{ Random 7} & \multicolumn{2}{|c|}{ Random 10} \\
\hline & & F1 & F2 & $\mathrm{F} 1$ & F2 \\
\hline \multirow{3}{*}{ PSO-BP } & RMSE & 0.0172 & 0.0218 & 0.0211 & 0.0209 \\
\hline & $\mathrm{R}^{2}$ & $98.92 \%$ & $98.27 \%$ & $98.37 \%$ & $98.41 \%$ \\
\hline & Time/s & 29.37 & 31.53 & 26.89 & 28.37 \\
\hline \multirow{3}{*}{ SVM } & RMSE & 0.0238 & 0.0242 & 0.0254 & 0.0224 \\
\hline & $\mathrm{R}^{2}$ & $97.90 \%$ & $97.83 \%$ & $97.6 \%$ & $98.12 \%$ \\
\hline & Time/s & 21.29 & 26.99 & 12.06 & 14.42 \\
\hline
\end{tabular}

\subsection{Experimental results}

Fig. 7(a)-(d) shows the comparison of actual coke amount and predicted values based on the PSO-BP algorithm when Random 7 and Random 10 were chose as datasets. It demonstrates that the selected feature sets and the established model can accurately predict the coke amount.

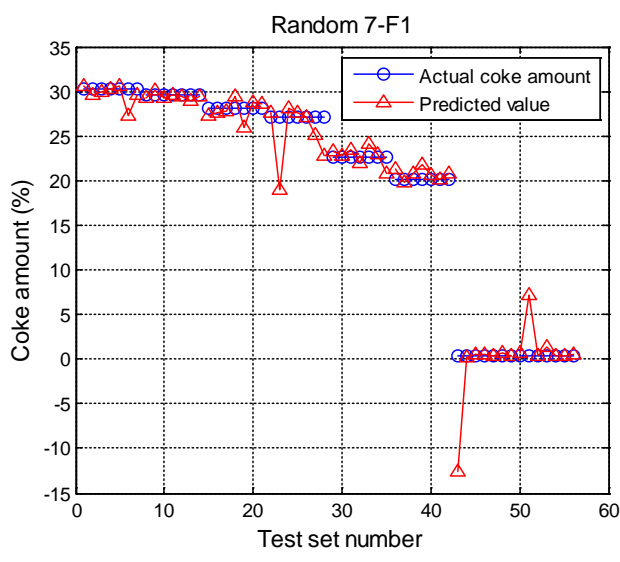

(a) Prediction results for Random 7 regarding F1.

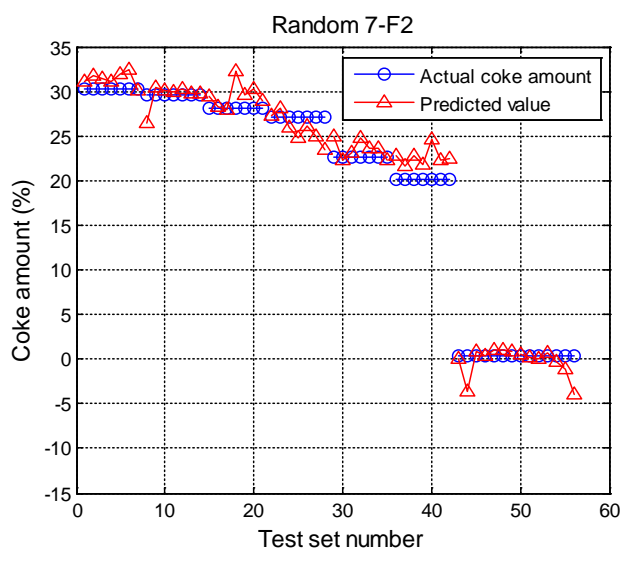

(b) Prediction results for Random 7 regarding F2. 


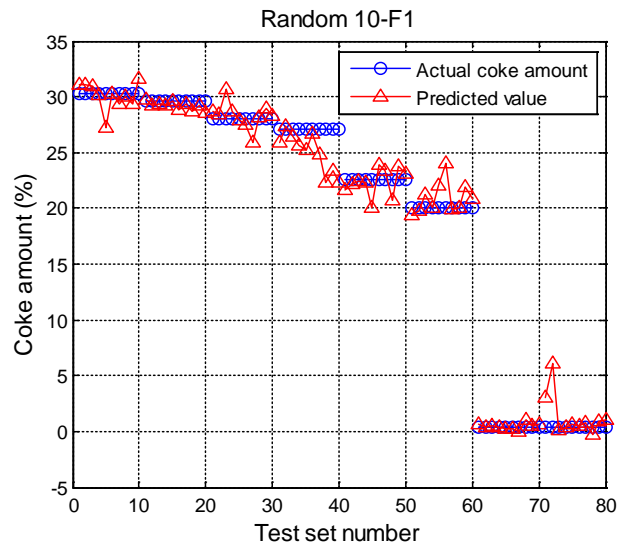

(c) Prediction results for Random 10 regarding F1.

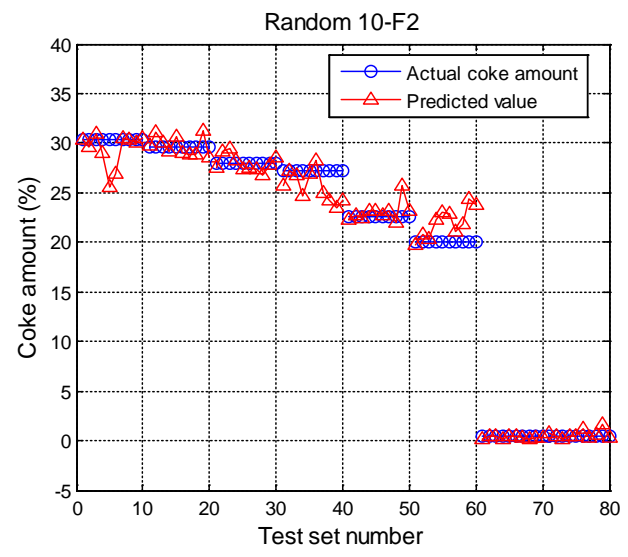

(d) Prediction results for Random 10 regarding F2.

Fig. 7. Comparison of the actual coke amount and predicted value of catalyst samples by PSO-BP with datasets of Random 7 and

$$
\text { Random } 10 .
$$

Furthermore, a set of validation experiments were conducted to verify the predicting ability of the prediction model to unknown samples based on various datasets. Three new datasets are obtained as follows: Class $0.448 / 20 / 28$ consists of three types of pellets whose coke amount are $0.448 \%, 20.067 \%$ and $28.038 \%$, and there are $75(25 * 3)$ pellets to be selected as the testing dataset, the left $125(25 * 5)$ ones as training dataset. Class 20/28 contains two types of pellets whose coke amount are $20.067 \%$ and $28.038 \%$ and there are $50(25 * 2)$ pellets chose as the testing dataset, the left $150(25 * 6)$ ones as training dataset. Class 27/0.448 is two types of pellets whose coke amount are $27.157 \%$ and $0.448 \%$. Fig. 8(a)-(f) display the prediction results of catalyst samples based on the PSO-BP algorithm, whose datasets are Class $0.448 / 20 / 28$, Class 20/28, Class 27/0.448 and feature sets are F1 or F2 respectively. The results prove the PSO-BP prediction model is able to classify unknown types of catalyst samples and predict the coke amount successfully.
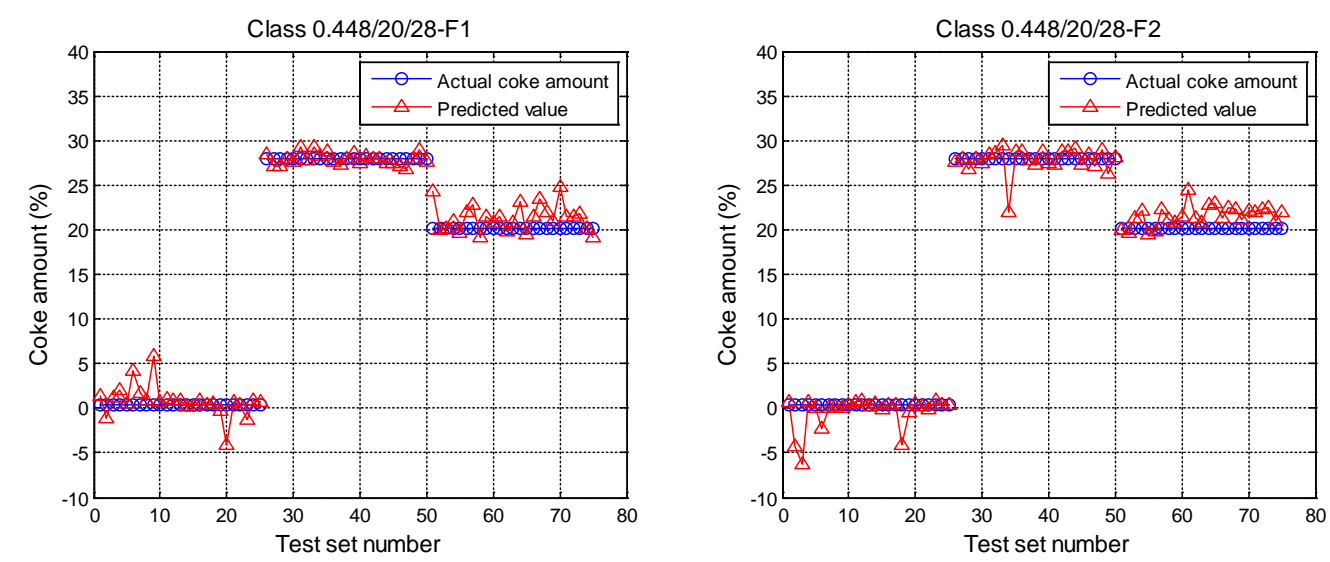


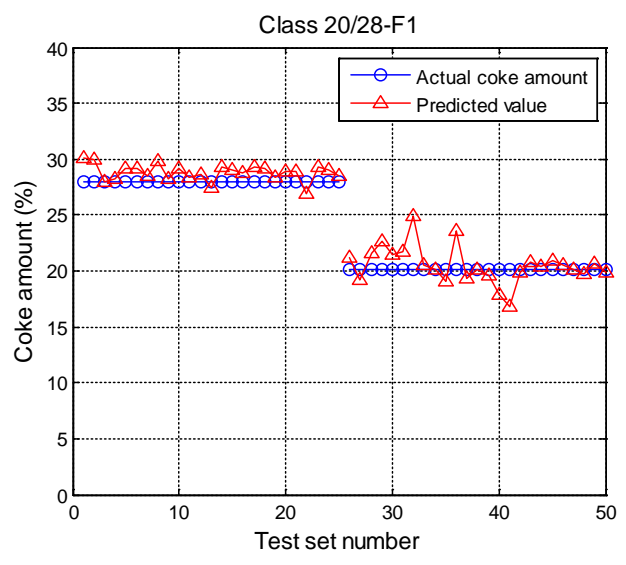

(c)Prediction results for Class 20/28 regarding F1.

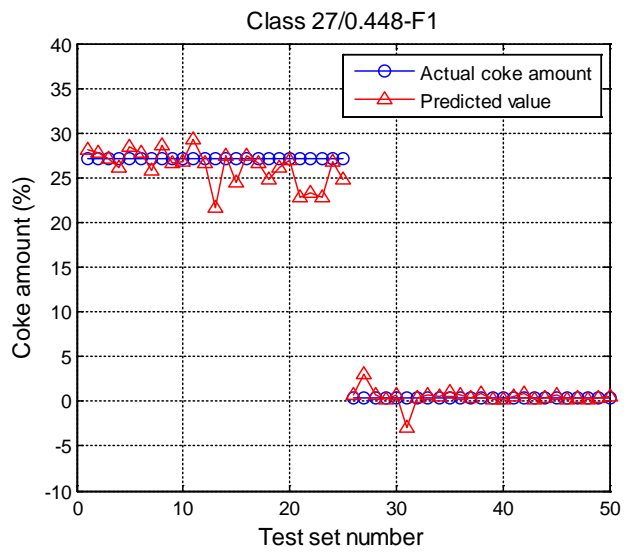

(e) Prediction results for Class 27/0.448 regarding F1.

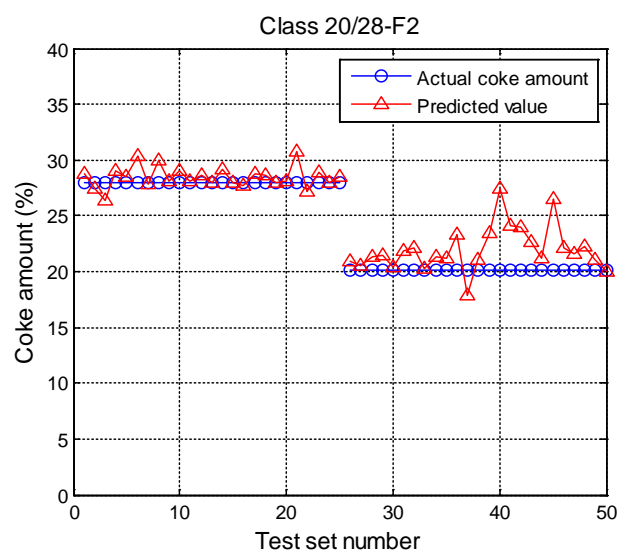

(d) Prediction results for Class 20/28 regarding F2.

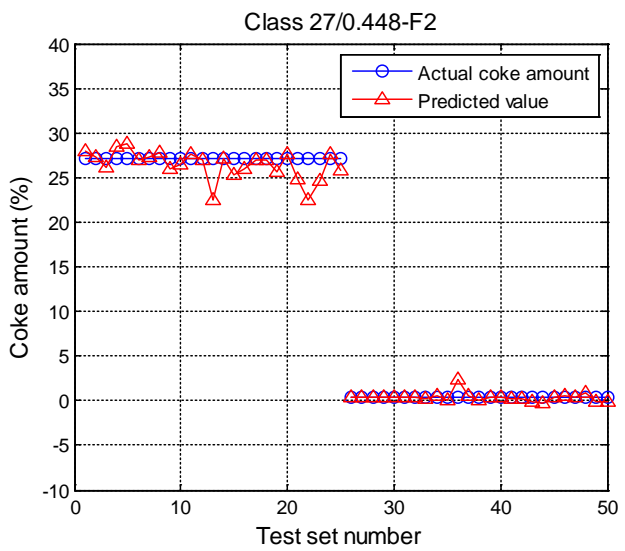

(f) Prediction results for Class 27/0.448regarding F2.

Fig. 8. Comparison of the actual coke amount and predicted value of catalyst samples by PSO-BP with datasets of Class 0.448/20/28, Class 20/28 and Class 27/0.448.

TABLE 6 Prediction Results for Different Datasets Regarding F1/F2 Based on PSO-BP

\begin{tabular}{|c|cc|cc|cc|}
\hline Index & \multicolumn{2}{|c|}{ Class $0.448 / 20 / 28$} & \multicolumn{2}{c|}{ Class 20/28 } & \multicolumn{2}{c|}{ Class 27/0.448 } \\
\hline RMSE & F1 & F2 & F1 & F2 & F1 & F2 \\
\hline $\mathrm{R}^{2}$ & 0.0157 & 0.018 & 0.0142 & 0.021 & 0.0167 & 0.0128 \\
\hline
\end{tabular}

Table 6 shows the prediction results for different datasets regarding F1/F2 based on PSO-BP algorithm. The RMSE of prediction values are all below 0.021 and the coefficient of determination $\mathrm{R}^{2}$ for the model are all above $78.71 \%$. The results indicate that the PSO-BP prediction model is precise for measuring the amount of coke deposition from different datasets. Thus the feasibility and effectiveness of this method based on image analysis and BP neural network to measure the amount of coke deposition on catalyst can be demonstrated.

\section{Conclusions}

This paper has proposed a new method through image analysis and soft computing to predict the amount of coke deposition on catalyst. A prediction model has been built based on the features from the image analysis using PSO-BP algorithm. The performance of the prediction model has been validated using various datasets. It may take some time to sample and handle the catalyst, depending on the 
actual process system. However, the data processing requires only about 1 to 2 minutes in this research, including image processing, model establishment and prediction. In future applications the data processing time will depend on the number of samples and performance of the computing processor. Although the current research is static, i.e. the data were collected and processed off-line, a potential application of this method is to achieve real-time measurement of coke deposition based on on-line sampling and continuous image analysis. The following conclusions are drawn from the results presented:

a) A flatbed scanner with an opaque cover has been found to be a simple and effective means to acquire high-quality images of catalyst samples.

b) The image processing algorithms developed are effective to identify correctly the regions of catalyst pellets.

c) The gray layer has been found to be the most effective colour layer for extraction of the colour features. Eleven colour features and six GLCM texture features are extracted from the processed images of catalyst pellets. Finally, twelve features are selected as the effective features and two best feature sets F1, F2 are obtained from the test results.

d) PSO-BP and SVM are used to establish the prediction model of coke amount when F1, F2 are taken as the feature sets respectively. The comparison results have shown that the PSO-BP algorithm outperformed the SVM algorithm. The prediction results have demonstrated that the PSO-BP model is precise for measuring the coke deposition amount for the datasets. The RMSE of prediction values are all below 0.021 and the $\mathrm{R}^{2}$ of the model are all above $78.71 \%$.

\section{Acknowledgements}

We would to thank the National Natural Science Foundation of China (No. 61403138), the Chinese Ministry of Science and Technology (No. 2012CB215203) and the Chinese Ministry of Education (No. B13009). This work is also supported by the Fundamental Research Funds for the Central Universities from North China Electric Power University (No. 2014QN13).

\section{References}

[1] Gross E, Liu J H C, Toste F D and Somorjai G A 2012 Control of selectivity in heterogeneous catalysis by tuning nanoparticle properties and reactor residence time Nature chemistry 4(11) 947-952

[2] Ren X H, Bertmer M, Stapf S, Demco D E, Blümich B, Kern C and Jess A 2002 Deactivation and regeneration of a naphtha reforming catalyst Appl. Catal. A: General 228(1) 39-52

[3] Aguayo A T, Sánchez del Campo A E, Gayubo A G, Tarrío A and Bilbao J 1999 Deactivation by coke of a catalyst based on a SAPO - 34 in the transformation of methanol into olefins Journal of Chemical Technology and Biotechnology 74(4) 315-321

[4] Wood J and Gladden L F 2002 Effect of coke deposition upon pore structure and self-diffusion in deactivated industrial hydroprocessing catalysts Appl. Catal. A: General 249(2) 241-253

[5] Chen D, Rebo H P, Moljord K and Holmen A 1997 The role of coke deposition in the conversion of methanol to olefins over SAPO-34 Studies in Surface Science and Catalysis 111 159-166

[6] Dejaifve P, Auroux A, Gravelle P C and Védrine J C 1982 Methanol conversion on acidic ZSM-5, offretite, and mordenite zeolites: A comparative study of the formation and stability of coke deposits Journal of Catalysis 70(1) 123-136

[7] Bibby D M, Milestone N B, Patterson J E and Aldridge L P 1986 Coke formation in zeolite ZSM-5 Journal of Catalysis 97(2) 493-502 
[8] Sun Z, Gu X and Yu J 2001 Coking models of reactor in continuous catalytic reforming Journal of East China University of Science and Technology 27(5) 568-571

[9] Bai Z W 2000 Determination of the components of mixture by thermogravimetric analysis Journal of Instrumental Analysis 19(4) $83-85$

[10] Li B H and Gonzalez R D 1998 The measurement of small amounts of coke by a sensitive TGA/FTIR technique Catalysis letters 54(1-2) 5-8

[11] Wang J A, Chen L F, Li C L and Novaro O 2001 Characterization of structure and combustion behavior of the coke Formed on a hydroisomerization catalyst Studies in Surface Science and Catalysis 139 53-60

[12] Bayraktar O and Kugler E L 2003 Coke content of spent commercial fluid catalytic cracking (FCC) catalysts Journal of thermal analysis and calorimetry 71(3) 867-874

[13] Tang Y Q, Cao Y J, Wang J D, Yang Y R, Jiang B B and Liao Z W 2011 A model for determination of amount of coke deposit on catalyst based on vibration signal analysis Chinese Journal of Mechanical Engineering 1 78-84

[14] Tang Y Q,Wang J D, Liao Z W, Yang Y R and Xie Z K 2010 Measurement of the amount of coke deposit on catalyst based on acoustic emission frequency shift Acta Petrolei Sinica (Petroleum Processing Section) 6 917-921

[15]Zhang J Q, Zhang W B and Yan Y 2015 Coke deposition detection through the analysis of catalyst images Proc. IEEE Int. Conf. on Imaging Systems and Techniques (Macau, China, 16-18 September 2015) pp 16-20

[16] Otsu N 1979 A threshold selection method from gray-level histograms IEEE Trans. on Systems, Man and Cybernetics 9(1) 62-66

[17] Stricker M and Orengo M 1995 Similarity of color images Proc. SPIE Int. Conf. on Electronic Imaging: Science and Technology (San Jose, CA, 5 February 1995) pp 381-392

[18] Zhang Z L, Yang J G,Wang Y L, Dou D Y and Xia W C 2014 Ash content prediction of coarse coal by image analysis and GA-SVM Powder Technology 268 429-435

[19] Mitani Y and Hamamoto Y 2010 A consideration of pan-sharpen images by HSI transformation approach Proc. 2010 Annual Conf. of the Society of Instrument and Control Engineers (Taipei, Taiwan, 18-21 August 2010) pp 1283-1284

[20] Mei Y and Androutsos D 2008 Color texture retrieval using wavelet decomposition in the independent components color space Proc. IEEE Int. Conf. on Electrical and Computer Engineering (Niagara Falls, Canada, 4-7 May 2008) pp 1379-1382

[21] Haralick R M, Shanmugam K and Dinstein I H 1973 Textural features for image classification IEEE Trans.on Systems, Man and Cybernetics 6 610-621

[22] Honeycutt C E and Plotnick R 2008 Image analysis techniques and gray-level co-occurrence matrices (GLCM) for calculating bioturbation indices and characterizing biogenic sedimentary structures Computers \& Geosciences 34(11) $1461-1472$

[23] Gonzalez R C andWoods R E 2007 Digital Image Processing 3rd (USA: Pearson Education)

[24] Anderson J A 1995 An Introduction to Neural Networks (MA: MIT Press)

[25] Chen F F, Jiang X F and Jiang Z Y 2011 The research of recognition on oceanic internal waves based on gray gradient co-occurrence matrix and BP neural network Proc. IEEE Int. Symposium on Photonics and Optoelectronics (Wuhan, China, 16-18 May 2011) pp 1-4

[26] Shi Y and Eberhart R C 1999 Empirical study of particle swarm optimization Proc. IEEE Int. Congress on Evolutionary Computation (Washington D.C., USA, 6-9 July 1999) pp 3

[27]Hsu C W and Lin C J 2002 A comparison of methods for multiclass support vector machines IEEE Trans. on Neural Networks 13(2) 415-425 